\title{
Arbor
}

\section{Ayudas ergogénicas en el deporte}

\section{Jose María Odriozola Lino}

Arbor CLXV, 650 (Febrero 2000), 171-185 pp.

Cuando se buscan los límites en la capacidad física de los humanos, toda mejora lleva implícita la optimización del funcionamiento del organismo. Para conseguirla se recurre a ayudas ergogénicas, aunque muchas de las utilizadas no se ha probado científicamente que consigan el efecto deseado. Además las hay permitidas y prohibidas (dopantes), saludables e insalubres.

Con arreglo a sus efectos las podemos agrupar en nutricionales y no nutricionales. Entre las primeras, estarían todos los suplementos dietarios, tales como vitaminas, minerales, bebidas y nutrientes energéticos. Entre las segundas, las ayudas mecánicas, psicológicas, fisiológicas y farmacológicas. Estas últimas incluyen el amplio grupo de sustancias prohibidas, que constituyen dopaje.

Las ayudas ergogéniras hacen más efecto, en general, a los deportistas recreacionales que a la élite y en todo caso la respuesta es siempre individual.

Para llegar a la elite en cualquier deporte, hacen falta normalmente muchos años de dedicación y constancia en los entrenamientos, además de unas facultades innatas adecuadas. Los deportistas intentan alcanzar el máximo rendimiento de sus condiciones físicas y adaptar metabólicamente su organismo para conseguirlo. Estos procesos anatomicofisiologicos son lentos de desarrollar y pueden sufrir interrupciones involuntarias debidas a errores en la metodología seguida o a lesiones o enfermedades imprevistas. Por otro lado, la mayór profesionalización de los deportistas, con grandes premios económicos y posibilidades de 
mejora social como estímulos, hacen que las metas a alcanzar sean mas apetecibles y se caiga en la tentación de que el fin justifique todos los medios, lícitos o no, para conseguirlo.

La llamada preparación biológica o farmacológica del deportista puede tener entonces dos facetas: contribuir de forma sensata y legal a que los atletas complementen adecuadamente sus entrenamientos para obtener un mejor rendimiento, añadiendo a su alimentación productos necesarios para mantener el alto ritmo metabólico inherente a esa dedicación de su organismo al esfuerzo físico, o intentar «atajos» para llegar en menos tiempo a la elite deportiva, con tratamientos farmacológicos o métodos artificiales prohibidos que mejoren su capacidad de alcanzar un mayor rendimiento físico. En ambas entra de lleno la ergogenia.

\section{¿Qué es la ergogenia?}

Este vocablo viene de las palabras griegas, ergon = trabajo y gennan = producción, es decir engloba todo lo relacionado con los mecanismos de producción de trabajo físico. Por ello, dentro de ayudas ergogénicas se pueden incluir todas aquellas sustancias, métodos, fármacos, equipamientos, máquinas, etc., que contribuyan a mejorar la capacidad innata para la producción o generación de trabajo físico por el organismo, generalmente de un deportista.

La primera y mas obvia es la que se obtiene como consecuencia del entrenamiento, que mejora las condiciones naturales heredadas por cada persona, siempre que sea adecuado y que vaya acompañado de una nutrición sana y equilibrada y un descanso reparador suficiente.

Pero existen otras ayudas para lograr esas superaciones por métodos mas artificiales y casi nunca recomendables. Sustancias que se dice pueden influir en la obtención o el ahorro de la energía necesaria para lograr mejores resultados deportivos. De muchas de ellas, la comunidad científica aun duda de su eficacia real para obtener ese objetivo y en cualquier caso no hay que olvidar la individualidad de cada persona, que condiciona que lo que a unos pueda sentar bien, a otros no les afecte o lo haga negativamente. Además se sabe que cuanto mas adaptado metabólicamente esté el cuerpo de un deportista a un determinado tipo de esfuerzo, menor será el posible efecto ergogénico de cualquier sustancia sobre él. 


\section{¿Qué efectos se buscan con las ayudas ergogénicas?}

De forma global, un aumento en la capacidad física de la persona y por tanto una mejora en su potencial para competir en cualquier deporte. Ello se puede conseguir fundamentalmente por tres vías: optimizando su capacidad de producir energía metabólicamente, incrementando la capacidad funcional de la maquinaria muscular y mejorando la capacidad competitiva.

La producción de energía para el esfuerzo físico proviene del consumo interno de materiales orgánicos capaces de liberarla. Fundamentalmente de las grasas e hidratos de carbono, almacenados o ingeridos por nuestro cuerpo, y en menor medida de las proteínas. Hay otros nutrientes que aunque no generan energía de si mismos, hacen posible la realización de los procesos metabólicos que la liberan de los compuestos energéticos: son las vitaminas, los minerales y el agua. Tanto unos como otros forman parte de los alimentos naturales que deben tomarse en cantidades adecuadas para que se pueda obtener el máximo rendimiento de esos procesos energéticos. También es necesario tomar diariamente una cierta cantidad de fibra para la correcta eliminación de los productos de desecho intestinales.

Para intentar mejorar la producción de energía que permita llevar a cabo un trabajo físico optimo, se pueden utilizar ayudas ergogénicas que mejoren la capacidad de aumentar las reservas energéticas, que activen los sistemas de movilizarlas al máximo y con la mayor celeridad posible y que contribuyan a una pronta y total reposición o regeneración de las mismas tras su consumo.

También podrán utilizarse para incrementar la capacidad funcional de la maquinaria muscular para, estando bien abastecida de energía, generar mayor cantidad de trabajo físico que permita mejores logros deportivos. Una hipertrofia de la masa magra muscular produce un aumento de fuerza que es la cualidad mas importante para mejorar en cualquier actividad deportiva.

También hay que adquirir mayor potencia aeróbica, así como conseguir recuperarse mas rápido de los esfuerzos intensos, evitando o disminuyendo la acumulación de subproductos del metabolismo energético, que contribuyen a la aparición de la fatiga y a una menor funcionalidad muscular y orgánica. Pueden asimismo producir una rebaja en el porcentaje de grasa corporal, eliminando peso innecesario.

Será también importante poder utilizar equipamientos que ayuden a obtener una mejor eficacia biomecánica, rebajando con ello el gasto energético. 
Por ultimo, la capacidad competitiva es fundamental para obtener buenos resultados y hay formas de estimularla, aunque muchas de ellas están prohibidas.

\section{Categorías de ayudas ergogénicas}

Podemos diferenciar cuatro posibles agrupamientos de las ayudas ergogénicas.

1. Las que se consideran legales, porque su uso está permitido por los máximos organismos deportivos internacionales y que en condiciones de utilización normal y dosis adecuadas, se sabe que no son peligrosas o perjudiciales para la salud del consumidor.

2. Las que no estando prohibidas, al menos de momento, sin embargo parece que pueden constituir un peligro potencial para la salud del que las utilice habitualmente.

3. Las que aun estando prohibidas por los organismos deportivos internacionales si son usadas adecuadamente no parecen constituir un peligro potencial para la salud del consumidor habitual.

4. Las que además de estar específicamente prohibidas y que por tanto ocasionarían sanciones, se sabe que son perjudiciales para la salud.

Por tanto vemos que aquí se entrecruzan y anteponen varios conceptos importantes para estas distintas categorías de ayudas ergogénicas. Por una parte, se tiene en cuenta como factor prioritario, el que por mejorar la capacidad física de una persona, no se esté poniendo en peligro su salud presente o futura. Pero también se valora el hecho de intentar jugar con ventaja con respecto a otros deportistas, al superar las capacidades físicas mejoradas solo por el entrenamiento de unos, con la utilización de métodos ergogénicos artificiales al alcance de unos pocos. Entraríamos de lleno en lo que se conoce por dopaje. Este vocablo, es la palabra española para «doping», que aunque inicialmente venía de «dope», narcótico o droga opiácea, siendo «doping» el hecho de estar narcotizado o drogado con productos psicotrópicos, extendió luego su denominación para incluir los efectos de otras sustancias que no actúan sobre el sistema nervioso, pero que estimulan artificialmente otras capacidades corporales del deportista.

El dopaje, constituye un problema ético, porque está en contraposición total con los principios básicos del «juego limpio», de competir sin hacer trampas a los contrarios, con ventajas fraudulentamente adquiridas. Es además un problema moral, porque va contra los prin- 


\section{Ayudas ergogénicas en el deporte}

cipios de la terapia médica, que se basa en recetar o aconsejar la toma de fármacos $\mathrm{u}$ otros productos que ayuden a prevenir ${ }^{\circ} \mathrm{o}$ curar enfermedades, no para ser usados por gente sana para mejorar sus marcas deportivas. Por último, constituye también un problema médico, porque altas dosis de fármacos potentes ingeridas por gente sana, pueden ocasionar efectos secundarios patogénicos graves.

Es obvio que el dopaje constituye un camino erróneo para llegar a formar parte de la elite deportiva. Todos debemos trabajar para conseguir su erradicación al máximo posible del mundo del deporte, no solo con métodos disuasorios basados en la realización de numerosos controles dentro y fuera de las competiciones, acompañados de sanciones ejemplarizantes cuando los resultados sean incontestables, sino también educando a los deportistas en esta materia. Informándoles de los tipos de dopaje que existen, de sus efectos secundarios insalubres y de los métodos alternativos y fármacos autorizados que pueden curarles y capacitarles para obtener buenos resultados deportivos. Que no se acostumbren a suplementarse por rutina. Que no se les recomienden productos solo por el efecto placebo. Que se recete cuando realmente se ha comprobado alguna carencia en su organismo pero siempre que sean fármacos permitidos o que no vayan a perjudicar su salud.

\section{Tipos de ayudas ergogénicas.}

Con el auge del mundo del deporte y de sus estímulos económicos y sociales, se ha disparado la comercialización de un gran número de ayudas ergogénicas. Muchas consisten en la mejora de los aparatos, instrumentos y equipamientos de los deportistas. Otras en técnicas de ayudas psicológicas, tan necesarias por las tensiones de todo tipo que origina la alta competición. Las hay de tipo fisiológico, para optimizar la buena funcionalidad metabólica general del organismo. El grupo mas numeroso y que evoluciona mas rápidamente es el de las ayudas farmacológicas, que incluyen una enorme variedad de productos permitidos y de productos dopantes. Todas estas son ayudas ergogénicas no nutricionales. Pero también existe una enorme cantidad de suplementos nutricionales, que pretenden completar y mejorar la alimentación del deportista, optimizando su recuperación durante o tras los esfuerzos o aumentar sus reservas energéticas necesarias para afrontar competiciones extenuantes.

Desde el punto de vista científico, para muchos de estos tipos de ayudas ergogénicas, no se han demostrado las mejoras por su utilización 
que pretenden atribuirles los que las comercializan. Para otras en cambio se conoce una clara relación causa-efecto y su uso, siempre que estén permitidas, es muy recomendable para determinadas especialidades deportivas. En la tabla I, vemos una posible clasificación de los diversos tipos de ayudas ergogénicas mas utilizadas en la actualidad.

TABLA I

\section{TIPOS DE AYUDAS ERGOGENICAS}

A) NO NUTRICIONALES

1) MECANICAS: BIOMECANICA

Vestimentas, cascos, bicis ,pistas sintéticas, esquis, zapatillas etc.

2) PSICOLOGICAS: PSICOLOGIA DEPORTIVA

Técnicas de concentración, de relajación, de perdida de ansiedad, etc. Hipnosis.

3) FISIOLOGICAS: FISIOLOGIA DEPORTIVA

Calentamiento, masaje, fisioterapia etc.

Autotransfusión sanguinea, inhalación de oxigeno.

4) FARMACOLOGICAS

a) PERMITIDAS:

$\begin{array}{ll}\text { Carnitina } & \text { Creatina } \\ \text { Bicarbonato sódico } & \text { Glucosamina } \\ \text { Citrato sodico } & \text { Ginsenosidos } \\ \text { Lecitina } & \text { Yohimbina } \\ \gamma \text {-Orizanol } & \text { Piruvato } \\ \text { Fosfatos } & \text { Antioxidantes } \\ \text { Glutation } & \text { Inosina } \\ \text { Colina } & \text { Ac. pangamico } \\ \text { DNA -RNA } & \text { Smilax }\end{array}$

b) PROHIBIDAS: DOPAJE

Estimulantes psico-motores (cocaina, pemolina, anfetaminas, cafeina) Analgesicos narcoticos (metadona, heroina)

Aminas simpatico-mimeticas (efedrina)

ANABOLIZANTES: Esteroideos (testosterona, estanozolol, mesterolona, nandrolona)

Peptidicos (EPO, GH, ACTH, HCG,IGF-1, insulina, factores de liberación hipotalamicos)

$\beta$-Bloqueantes

Otros (clembuterol)

Diuréticos, métodos enmascaradores (probenecid)

Anestésicos locales, corticosteroides, alcohol, cannabis.

B) NUTRICIONALES

1) SUPLEMENTACION CON MACRONUTRIENTES: gramos por día. 


\section{Ayudas ergogénicas en el deporte}

Bebidas deportivas-energeticas

Dieta de supercompensación de carbohidratos

Suplementos proteicos (hidrolizados, pastillas)

Suplementos con aminoacidos:

ARG, ORN, LYS, GLY (efectos anabolizantes)

Aminoácidos ramificados (LEU, ILE, VAL) para energía.

Polen abeja, jalea real, miel.

Algas (espirulina), levadura de cerveza

Gelatina (colágeno)

TG de cadena media, ácidos grasos de la serie w-3

2) SUPLEMENTACION CON MICRONUTRIENTES: miligramos o

microgramos por día.

a) VITAMINAS:

Complejo B, Antioxidantes (C, E, Carotenoides)

b) MINERALES:

Cromo, boro, magnesio, hierro, calcio, selenio, zinc.

\section{¿Qué posibles efectos tienen las ayudas ergogénicas más utilizadas?}

La finalidad de utilizar ayudas ergogénicas, para mejorar la capacidad física, va encaminada sobre todo a limitar los efectos fatigantes del ejercicio, tanto intenso como prolongado. Ello se puede lograr, principalmente por un aumento previo de la fuerza o capacidad muscular (hipertrofia muscular), como por iniciar el ejercicio con mayor cantidad de reservas energéticas (glucógeno) o de transportadores de oxígeno (glóbulos rojos). Durante el esfuerzo, se tratará de evitar la acumulación de factores fatigantes, tanto del metabolismo anaeróbico (en ejercicios de alta intensidad), tales como lactatos, acidificación muscular, etc., como del aeróbico (ejercicio prolongado y de resistencia), tales como deshidratación o hipoglucemia.

Vamos a comentar, escuetamente, las características de las ayudas ergogénicas permitidas, que son más frecuentemente utilizadas por muchos deportistas, indicando si los efectos positivos que se les atribuyen tienen base científica o no. De las dopantes no vamos a comentar más, pues no es el objeto de este trabajo y lo alargaría en exceso.

$M E C A N I C A S$. La Biomecánica es una ciencia de gran importancia en la mejora de las prestaciones deportivas. Son obvios los avances obtenidos en cuanto a resultados se refiere, como consecuencia de estudios biomecánicos en todo tipo de vestimenta y equipamiento deportivo. Los trajes aerodinámicos, cascos, esquís, bicicletas, zapatillas 
de clavos, que hoy día usan los deportistas, han posibilitado logros muy importantes en la consecución de nuevos récords. Las técnicas de entrenamiento, también se basan en aspectos biomecánicos, como el ángulo de salida de un lanzamiento o la posición del ciclista sobre la bicicleta, por ejemplo.

PSICOLOGICAS. Hoy día, la presión que sufren los deportistas en la alta competición es muy grande. Continuamente se les originan tensiones, que deben ser correctamente superadas. Existen técnicas para concentrarse mejor, relajarse si es necesario o incluso aumentar la agresividad ante la competición, las ansias de triunfar. A ello ayuda la Psicología deportiva, optimizando la mentalidad del competidor y mejorando sus resultados.

FISIOLOGICAS. De todos es conocida la importancia de un buen calentamiento, para poner todo el metabolismo energético en condiciones de funcionar a su máxima capacidad en el momento del esfuerzo competitivo, así como elevar la temperatura corporal necesaria para optimizar las reacciones enzimáticas. Asimismo, el masaje previo o posterior a las pruebas contribuye a un mejor rendimiento o recuperación.

En esfuerzos aeróbicos, la capacidad de transporte de oxigeno es fundamental y puede ser mejorada por transfusiones sanguíneas que aumentan la dotación de glóbulos rojos. Este es un método prohibido y se considera dopaje, aunque si se lleva a cabo correctamente no tiene porqué perjudicar la salud del deportista.

La inhalación de oxígeno en alta concentración, se ha demostrado que no mejora en nada la llegada del mismo al músculo y, por tanto, se considera un método inútil como ayuda ergogénica, aunque puede tener un cierto efecto placebo.

\section{FARMACOLÓGICAS:}

Carnitina: Es un metabolito transportador de ácidos grasos entre el citosol y las mitocondrias celulares donde estos son consumidos para generar energía. Se sintetiza por el organismo diariamente y tiene una presencia importante en el músculo cardiaco y esquelético.

Se le atribuyen muchos efectos positivos, tras su ingestión, aunque no hay nada científicamente demostrado: que favorece la pérdida de grasa, que mejora la resistencia en esfuerzos prolongados retrasando la aparición de fatiga y que reduce la sensación de dolor muscular, entre otros. Su toma, no parece ocasionar efectos secundarios. 
Creatina: Muy utilizada entre los deportistas de alto nivel. Este aminoácido nitrogenado no proteico se encuentra sobre todo en el músculo esquelético, donde proporciona energía (ATP) de gran calidad en el metabolismo anaeróbico alactácido. El problema es que solo dura unos pocos segundos durante un esfuerzo intenso, pues su almacenamiento es limitado (un $75 \%$ en forma de creatina-fosfato y el resto como creatina libre). Un hombre de $70 \mathrm{~kg}$ de peso, puede acumular entre 120 y 140 grs.

Se sintetiza diariamente en el hígado, riñón y páncreas, a partir de los aminoácidos Arginina, Glicocola y Metionina, en una cantidad aproximada de 1-2 gramos por día. Con la dieta se ingieren alrededor de 1- 2 gramos, principalmente en alimentos de origen animal, carne y pescado, que contienen unos 20 gr de creatina por kilogramo. $\mathrm{Si}$ se aumenta la ingesta, se retroinhibe la síntesis endógena. Se excreta por el riñón, 1-2 gramos por día, en parte como creatinina, que es el producto terminal del catabolismo de la creatina.

Se han hecho muchas investigaciones para comprobar los posibles efectos positivos de la suplementación de creatina. Lo comprobado, hasta el momento, podemos resumirlo de la siguiente manera:

- La ingestión de 20-25 grs./día de monohidrato de creatina (ó 0,3 g./Kg. Peso/día), durante 5-6 días, ocasiona un incremento de entre el 20 y el $30 \%$ de creatina en el músculo, de la que aproximadamente una quinta parte se almacena como creatina-fosfato. Estas dosis de «carga» serán más efectivas si se acompañan de bebidas con carbohidratos (90 gramos, 4 veces al día).

- Las dosis de «mantenimiento», se establece en unas 10 veces menos que la cantidad de "carga», por día.

- Si se aumenta esta suplementación de creatina, el músculo ya no retiene más y la que se toma en exceso será excretada por los riñones en la orina. La concentración máxima que se puede alcanzar en el músculo es de 150-160 milimoles por kilogramo.

- La ingesta de creatina parece recomendable a los vegetarianos, que pueden tener un déficit crónico, por la falta casi total de la misma en su dieta.

- Como efectos positivos sobre el rendimiento de deportistas se ha comprobado que puede mejorar el rendimiento en sesiones de ejercicio intermitente de alta intensidad (sprints en bicicleta estática o levantamiento de pesas), al retrasar la aparición de síntomas de fatiga, por la mayor disponibilidad inicial de creatina-fosfato, así como un mayor ritmo de resíntesis de la misma en los periodos de recuperación cortos. Se observa también una 
reducción de la concentración de lactato en sangre y una acumulación de hipoxantina, que indican un cambio en el origen de los productos consumidos para generar la energía (menor incidencia de la ruta anaeróbica lactácida). Sin embargo, hay datos contradictorios en trabajos llevados a cabo fuera del laboratorio, para esfuerzos de corta duración (carreras de velocidad, natación y ciclismo).

- No se han demostrado mejoras ni en el rendimiento ni en la capacidad máxima de consumo de oxígeno (aeróbica) en ejercicios prolongados e ininterrumpidos, de resistencia.

- Se ha publicado, que también puede producir un aumento en la síntesis de proteínas musculares, pero no está demostrado que haya una relación causa-efecto. Quizás hay hipertrofia muscular, al llevar a cabo más repeticiones de ejercicios de fuerza, durante los entrenamientos. Además parece que hay un cierto efecto «placebo», con la «sensación» de incremento en la capacidad muscular.

- Efectos secundarios: Se ha observado un aumento de peso (masa corporal) de entre 1 y $2 \mathrm{Kg}$, tras una suplementación de 20 grs./día, durante 5-6 días. Se cree que es debido a una mayor retención de agua en las fibras musculares. Eso será negativo para el rendimiento deportivo, sobre todo en esfuerzos prolongados.

Además, puede haber a la larga una mayor presión renal, por el exceso de creatina ingerida que no se almacena en los músculos y que debe ser excretada en la orina.

Como todas las teóricas ayudas ergogénicas, la respuesta es individual (se sabe que un 20-30\% de deportistas no retienen la creatina exógena) y debe probarse con precaución y con tiempo, antes de las competiciones importantes.

\section{Bicarbonato sódico o citrato sódico}

La base del posible efecto ergogénico del bicarbonato o del citrato sódico, es como tamponador (neutralizador) de la acidificación producida por el esfuerzo anaeróbico en los músculos, que produce fatiga y pérdida de eficacia en la contracción muscular.

Se ha comprobado que la ingestión 1-2 horas antes del ejercicio de 0,3 gr de bicarbonato por $\mathrm{kg}$ de peso corporal del deportista, en aproximadamente 1 litro de agua ( 21 gr para una persona de $70 \mathrm{Kg}$, o sea 4-5 cucharaditas de bicarbonato) produce ese efecto neutralizador 


\section{Ayudas ergogénicas en el deporte}

parcial de la bajada de pH, en esfuerzos de 1 a 7 minutos de duración. También podría favorecer el entrenamiento de «intervalos» (series cortas e intensas con poca recuperación entre ellas), así como reducir la percepción psicológica de fatiga en esfuerzos de alta intensidad.

No se ha visto en cambio que produzca ningún efecto positivo en esfuerzos más largos (aeróbicos). Asimismo, ese efecto ergogénico parece ser significativo en deportistas poco entrenados, pero en la elite ya su propia adaptación al ejercicio agonístico, les desarrolla sistemas enzimáticos y tamponadores endógenos, por lo que el bicarbonato no mejora sus prestaciones.

Como efectos secundarios, puede producir problemas gastrointestinales, tales como náuseas, dolor estomacal por la excesiva alcalinidad producida y diarreas. En grandes dosis y uso prolongado puede producir alcalosis severa con apatía e irritabilidad e incluso espasmos musculares y arritmias cardiacas.

\section{Ginsenosidos}

Sustancias obtenidas de las raíces del Ging-seng. Producen infusiones de sabor dulce y aromático, que parecen estimular al organismo en varias formas, incluyendo la de actuar como afrodisiacos. Todos esos efectos no están científicamente demostrados y la respuesta es muy individual y psicológica.

No se ha podido demostrar que realmente beneficien la capacidad física, ni retrasen la percepción de fatiga, ni la capacidad de recuperación tras esfuerzos agonísticos, como les atribuyen sus adeptos.

Como efectos secundarios y en cantidades importantes puede producir insomnio, nerviosismo, irritabilidad, hipertensión y diarreas, además de síndrome de abstinencia, al dejar de tomarlo tras su ingestión continuada por largos periodos.

\section{Glucosamina}

Principal precursor de los glicosaminoglicanos que junto con el colágeno son los componentes más importantes del tejido conectivo en los ligamentos y tendones. Por tanto es fundamental, tanto para su formación, como para su regeneración, tras lesiones por ejemplo. Una ruptura de cartilagos requiere una mayor síntesis de glucosamina para acelerar la cicatrización. La actividad de la enzima que la sintetiza, a partir de glucosa y glutamina, la glucosamina sintétasa, en por tanto el factor limitante para esa remodelación del tejido conectivo. Esa actividad declina con el envejecimiento. 
La suplementación con sulfato de glucosamina, en dosis que van desde $500 \mathrm{mg}$ a 6 gramos por día, dependiendo de la severidad del problema, parece subir los niveles de este compuesto en el tejido conectivo, hasta un $17 \%$, acelerando la curación de la lesión. De momento no se conocen posibles efectos secundarios de esta suplementación.

\section{Cafeina}

La cafeína, alcaloide que se encuentra en el café, en el cacao (chocolate) y en muchas bebidas refrescantes (colas), produce un efecto estimulante en el organismo y, por ello, está prohibida por encima de determinados niveles. (12 microgramos por mililitro de orina del deportista).

En dosis permitidas, parece acelerar la lipolisis, con el consiguiente ahorro inicial de glucógeno en esfuerzos prolongados, así como «alertar» el organismo con cierto grado de estimulación. En deportistas entrenados, parece no ser necesario, por la ya comentada adaptación al ejercicio, de su metabolismo.

En exceso puede producir nerviosismo y problemas gastrointestinales, además de diuresis.

\section{Yohimbina}

Es un alcaloide nitrogenado que se obtiene de la corteza del árbol yohimbe. Se sabe que bloquea como antagonista los receptores adrenérgicos del tipo $\alpha-2$, lo que provocaría un incremento de los niveles séricos de NA. Se suele usar en tratamientos contra la obesidad y la impotencia sexual. Se especula con que podría incrementar los niveles endógenos de testosterona, aunque no está demostrado.

\section{Lecitina}

Es un fosfolípido, la fosfatidilcolina, producido por el propio organismo. Supuestamente podría prevenir la acumulación de grasas.

\section{Piruvato}

En los años ochenta, se hicieron experiencias y se decía que 20-25 gramos de piruvato por día, parecía incrementar la resistencia y facilitar la pérdida de peso (?). Actualmente se han vuelto a hacer pruebas con voluntarios no deportistas y lo único que se ha comprobado es que bajan sus niveles de concentración de HDL en sangre. 


\section{Ayudas ergogénicas en el deporte}

$\gamma$-orizanol

Es un fitosterol que se extrae del arroz. Se le' atribuyen efectos como provocar un aumento de la testosterona y de la somatotropina (GH) en suero y que por ello podría provocar hipertrofia muscular. No hay nada científicamente demostrado. Algo parecido se dice del smilax, que también contiene fitosteroles.

\section{Antioxidantes}

Todo tipo de compuestos que contengan enzimas que bloquean los radicales libres que provocan oxidación celular. Por ejemplo, la superóxido dismutasa o la glutation peroxidasa. Si se toman por vía oral, al tratarse de proteínas, se digieren y destruyen antes de llegar a su posible destino.

\section{Inosina}

Es un nucleósido, que se utiliza en la síntesis de los nucleotidos fosfato, como el ATP. Por ello podría favorecerse su disponibilidad, con la consiguiente mejora de fuerza y resistencia. Pero no hay nada seriamente demostrado al respecto.

\section{Colina}

Componente aminado de algunos fosfolípidos, y concretamente de las lecitinas, así como de la acetilcolina que pone en marcha el mecanismo de la contracción muscular. El cuerpo la fabrica endógenamente y no se han probado sus supuestos efectos ergogénicos sobre la fuerza y la pérdida de grasa.

\section{Acido pangámico}

También llamado, durante los JJOO de Moscú en el 80, vitamina B15. Aumentaría, según sus promotores la «disponibilidad» de oxígeno en los esfuerzos aeróbicos aunque no lo han demostrado científicamente.

\section{NUTRICIONALES}

Cuantas más investigaciones se hacen sobre el posible efecto de suplementos nutricionales sobre el rendimiento o la salud de los deportistas, más se llega a la conclusión de que con una buena alimentación, suficiente en cantidad y equilibrada en calidad, no hace falta suplementación alguna. Asimismo se comprueba que el exceso en me- 
gadosis de algunos nutrientes, tipo vitaminas o minerales, no sólo no mejora la capacidad física del deportista, sino que puede incluso resultar perjudicial para su salud y por tanto para el rendimiento deportivo. Otra cosa es que la persona tenga déficits de algunos de ellos y la correspondiente ingestión de los mismos evite una patología y favorezca la normalización metabólica del paciente.

Por tanto, no se recomienda tomar rutinariamente o por placebo ningún tipo de suplemento nutricional, si previamente no se ha detectado el déficit o para prevenirlo, si el historial clínico del atleta así lo recomienda (caso del hierro o del calcio en determinados colectivos, sobre todo mujeres, y en épocas concretas).

Se ha comprobado sin embargo que determinadas dietas, con mayor porcentaje de algunos nutrientes, pueden resultar ergogénicamente beneficiosas para algunas especialidades deportivas. De los nutrientes energéticos, la proporción recomendada en la dieta, con referencia al total de calorías ingeridas diariamente, es que el 55-60\% lo deben ser en forma de carbohidratos, el $15 \%$ de proteínas y el resto (25-30\%) de grasas.

Uno de los problemas en los esfuerzos de larga duración (por lo menos 75-90 minutos de ejercicio intenso y continuado), es que se acaban terminando las reservas de glucógeno, que es el combustible muscular que proporciona una mayor capacidad de ritmo metabólico $\mathrm{y}$ por tanto un mejor rendimiento físico. Para retrasar esta causa de fatiga lo más posible, se recomienda hacer en los tres días anteriores al esfuerzo de larga duración (un maratón, por ejemplo) la llamada «dieta de supercompensación de carbohidratos». Esta consiste básicamente en subir el porcentaje de carbohidratos (mayoritariamente complejos) hasta el $70 \%$ diario y beber mucha agua. Con ello se puede hasta casi duplicar las reservas de glucógeno hidratado y retrasar la típica "pájara» que sobreviene cuando se acaban.

El beber durante los esfuerzos prolongados, agua o bebidas energéticas (con azúcares e incluso sales disueltas) puede evitar la deshidratación, que es el otro gran factor limitante y fatigante en muchas competiciones de resistencia. Cada deportista debe habituarse al tipo de bebidas que le van mejor y que no le ocasionan problemas grastrointestinales, tales como náuseas, vómitos, diarreas, etc. El agua fresca es, en todo caso, la más universal y positiva forma de retrasar la deshidratación, tomada en cantidades de 250-300 ml cada 15-20 minutos de esfuerzo continuo.

Todas las demás posibles suplementaciones nutricionales, tales como mezclas de aminoácidos, proteína en polvo, vitaminas y minerales, 


\section{Ayudas ergogénicas en el deporte}

entre las más usadas, ya se ha dicho que no se ha comprobado su efecto ergogénico y en exceso pueden incluso provocar efectos secundarios negativos para la salud del deportista.

\section{Bibliografía}

Balsom, P.D.; Ekblom, B.; Soderlund, K.; Sjodin, B. e Hyltman, E.: (1993a) Creatine supplementation and dynamic high- intensity intermittent exercise. Scand J.Med. Sci. Sport, 3, 143-149.

Balsom, P.D.; Harridge, S.D.R.; Soderlund, K.; SJodin, B. y Ekblom, B. (1993b): Creatine supplementation per se does not enhance endurance exercise performance. Acta Physiol. Scand., 149, 521-523.

Clarkson, P.M. (1991): Minerals: exercise performance and supplementation in athletes. J. of Sports Sci., 9, 91-116.

JuHN MARK, S. (1999): “Oral creatine supplementation» 47-58. The Physician and Sport Medic. 27, 5 .

Katch, F.J. y McArdle, W.D. (1993): Optimal nutrition for exercise and good health. En Katch and McArdle, Introduction to Nutrition, Exercise and Health 149-168. London. Lea-Febiger.

SPRIETT, L.L. (1997): Ergogenic aids: recent advances and retreats. In Lamb D.R., R. Murray (eds) Cooper, Carmel, 185-237.

VAN DER BEEK, E.J. (1991): Vitamin supplementation and physical exercise performance. J. of Sports Sci., 9, 77-89.

Williams, M.H. (1993): Nutritional supplements for strength trained athletes. Sport Sci. Exchange. Gatorade Sports Sci.Inst., 6, 6. 